\title{
Modeling phonotaxis in female Gryllus bimaculatus with artificial neural networks
}

\author{
Gundula Meckenhäuser ${ }^{1,2^{*}}$, Matthias R Hennig ${ }^{3}$, Martin P Nawrot ${ }^{1,2}$ \\ From Twentieth Annual Computational Neuroscience Meeting: CNS*2011 \\ Stockholm, Sweden. 23-28 July 2011
}

Courtship songs are used by female Gryllus bimaculatus to assess the quality of their potential mating partner. Typically, a song consists of chirps composed of few pulses followed by a chirp pause (Figure 1A). In extensive behavioral experiments, phonotactic responses of female crickets were tested by varying song features such as duration of pulse, pulse-pause, pulse-period, etc. [1]. Although several features of the song have been determined to affect the attractiveness of a song, the exact mechanisms underlying the female's evaluation process are yet unclear.

Here, we present a feed-forward artificial neural network that quantitatively predicts the attractiveness of mating songs. Our approach was motivated by recent work from Wittmann et al. [2] who presented a network that analyzes and evaluates courtship songs of grasshop- pers (Chorthippus biguttulus).We studied networks consisting of twelve neurons in the input layer, each one representing a feature of a given song, a variable number of $n=1, \ldots, 15$ neurons in the hidden layer and one output neuron that represents the phonotaxis. The neurons from one layer to the next are all-to-all connected via synaptic weights. The weights are trained with 160 artificial courtship songs for which the phonotaxis had already been determined in experiments. For training our networks, we used the backpropagation algorithm. We show that the mean squared error computed from a test set of 40 courtship songs is minimal for artificial neural networks with $\mathrm{n}=3$ hidden neurons. To estimate the predictive power of 3-hidden-neurons networks, we analyze the correlation between the model's phonotaxis and the female's phonotaxis. Figure 1B.

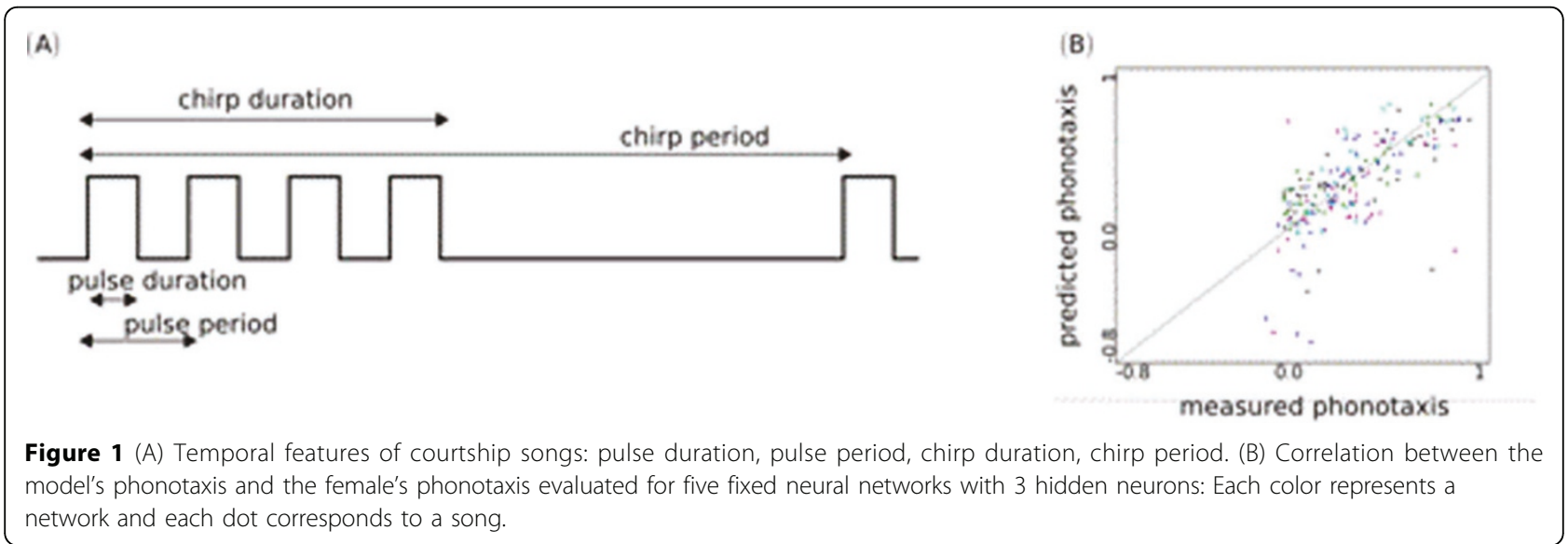

* Correspondence: gundula.meckenhaeuser@fu-berlin.de

${ }^{1}$ Freie Universität Berlin, Berlin, Germany

Full list of author information is available at the end of the article

(c) 2011 Meckenhäuser et al; licensee BioMed Central Ltd. This is an open access article distributed under the terms of the Creative 
shows phonotaxis values predicted by 3-hidden-neurons networks versus the values measured in experiments. Each color represents one fixed network. The best performing network yielded a mean squared error of 0.05 . Thus, our model can be used for a quantitative prediction of the attractiveness of untested courtship songs and so it complements experimental testing of female phonotaxis in the laboratory.

\section{Acknowledgement}

This work is funded by the German Research Council (DFG) within the Collaborative Research Center Theoretical Biology (SFB 618).

\section{Author details}

${ }^{1}$ Freie Universität Berlin, Berlin, Germany. ${ }^{2}$ Bernstein Center for Computational Neuroscience Berlin, Germany. ${ }^{3}$ Behavioural Physiology Group, Department of Biology, Humboldt-Universität zu Berlin, Berlin, Germany.

Published: 18 July 2011

\section{References}

1. Hennig RM: Walking in Fourier's space: algorithms for the computation of periodicities in song patterns by the cricket Gryllus bimaculatus. J Comp Physiol A 2009, 196:971-987.

2. Wittmann JP: A neural network-based analysis of acoustic courtship signals and female responses in Chorthippus biguttulus grasshoppers. J Comput Neurosci 2010.

doi:10.1186/1471-2202-12-S1-P234

Cite this article as: Meckenhäuser et al:: Modeling phonotaxis in female Gryllus bimaculatus with artificial neural networks. BMC Neuroscience 2011 12(Suppl 1):P234.

\section{Submit your next manuscript to BioMed Central} and take full advantage of:

- Convenient online submission

- Thorough peer review

- No space constraints or color figure charges

- Immediate publication on acceptance

- Inclusion in PubMed, CAS, Scopus and Google Scholar

- Research which is freely available for redistribution

Submit your manuscript at www.biomedcentral.com/submit
C Biomed Central 